Biol. Cybern. 71, 489-501 (1994)

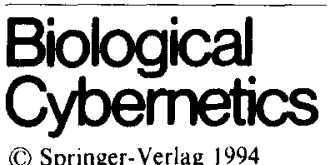

\title{
Frequency dependence of the action-perception cycle for postural control in a moving visual environment: relative phase dynamics
}

\author{
T. M. H. Dijkstra ${ }^{1}$, G. Schöner ${ }^{2}$, M. A. Giese ${ }^{2}$, C. C. A. M. Gielen ${ }^{1}$ \\ ${ }^{1}$ Laboratory of Medical Physics and Biophysics, University of Nijmegen, P.O. Box 9101, NL-6500 HB Nijmegen, The Netherlands \\ ${ }^{2}$ Institut für Neuroinformatik, Ruhr-Universität Bochum, Postfach 102148, ND04, D-44780 Bochum, Germany
}

Received: 7 September 1993/Accepted in revised form: 2 May 1994

\begin{abstract}
When standing human subjects are exposed to a moving visual environment, the induced postural sway displays varying degrees of coherence with the visual information. In our experiment we varied the frequency of an oscillatory visual display and analysed the temporal relationship between visual motion and sway. We found that subjects maintain sizeable sway amplitudes even as temporal coherence with the display is lost. Postural sway tended to phase lead (for frequencies below $0.2 \mathrm{~Hz}$ ) or phase lag (above $0.3 \mathrm{~Hz}$ ). However, we also observed at a fixed frequency, highly variable phase relationships in which a preferred range of phase lags is prevalent, but phase jumps occur that return the system into the preferred range after phase has begun drifting out of the preferred regime. By comparing the results quantitatively with a dynamical model (the sine-circle map), we show that this effect can be understood as a form of relative coordination and arises through an instability of the dynamics of the action-perception cycle. Because such instabilities cannot arise in passively driven systems, we conclude that postural sway in this situation is actively generated as rhythmic movement which is coupled dynamically to the visual motion.
\end{abstract}

\section{Introduction}

The stabilisation of posture has been intensely studied over the last few decades, not only because of its clinical relevance, but also because it can serve as a model system for multisensory integration. A prominent approach has been to look at the contribution of various sensory systems to the reduction of sway amplitude and to the temporal structure of sway. In particular, the role of visual information has thus been analysed, and it was found that visual information stabilises posture under normal conditions (Dichgans and Brandt 1978), but may destabilise posture if brought into conflict with the

Correspondence to: C. C. A. M. Gielen stationary environment as sensed by other sensory channels (Lee and Lishman 1975; Lestienne et al. 1977). By varying the spatiotemporal structure of visual information, the response properties of the postural system have been identified (van Asten et al. 1988a, b).

In recent theoretical and experimental work (Schöner 1991; Dijkstra et al. 1994) we showed that the postural control system can be characterised by a dynamical model based on variables that express the functional state of the complete system, in our case the position of the eye in the inertial world. From the point of view of the nervous system, such variables belong to a highly integrated level of description because no single sensory or motor system provides information on these variables directly. However, the postural control system can be described by such variables by mapping observed behaviour (upright standing) onto attractor solutions of dynamical systems. The experimental work, in which the distance of a moving visual scene from the observer was varied, confirmed predictions from a dynamical model based on this approach. However, important deviations from the mathematical form of the model (linear driven oscillator) were observed, which were interpreted as indicative of an active contribution of the postural control system in response to visual motion.

In this article we report experimental and modelling work that aimed to look afresh at the problem of how visual information is integrated into posture behaviour. We went back to the paradigm of characterising the properties of the postural system to visual stimulation by varying the frequency of sinusoidal visual motion (Lestienne et al. 1977; Talbott 1980; van Asten et al. 1988a). Based on concepts from linear systems theory, these studies aimed to characterise the response properties of the postural control system. Linear systems theory was critically evaluated by Talbott and coworkers studying postural stability in dogs (Schuster and Talbott 1980; Talbott 1980; Talbott and Brookhart 1980). From a series of experiments in which dogs stand on a movable table and are exposed to a moving visual environment, these authors concluded that the influence of vision on posture is strongly task-dependent, a conclusion in line 
with our present concerns. However, while gain was shown to be strongly non-linear in response to both table motion and visual motion, no clear non-linearities in response to visual motion alone were found. Also, they never found any significant response at frequencies other than those used for input. Our aim is to challenge the view in these studies that the postural system is passively driven by visual motion. Another way to put this is to ask if the influence of visual motion on posture relies on the existence of a coherent temporal relationship between postural sway and visual motion. If posture is essentially a passive control system driven by visual motion (or other sensory channels), then postural sway must reflect the time structure of sensory input whenever such input affects posture. If, on the other hand, postural sway is actively generated based on perceived conditions, then input may affect posture even as temporal coherence is lost. Specifically, in the active case, periodic visual motion may induce periodic sway of adequate amplitude even if sway and visual motion are not phase-locked.

To answer this question, we have developed techniques which enable us to analyse the system in a regime where coherence between visual information and postural sway is lost. These techniques rely on the extraction of a relative phase time series as a measure of the temporal structure of the action-perception cycle. These time series are analysed with respect to stability and dynamic properties through return maps and histogram techniques. Furthermore, a concrete model (stochastic sinecircle map) is fitted to the relative phase data in various ways. The stochastic sine-circle map has a dynamics which is rich enough to capture both phase-locked (linear) and not locked (non-linear) behaviour. We will show that we can reliably fit the stochastic sine-circle map to our relative phase data. In addition, more common frequency domain methods are applied as well.

We exposed standing subjects to a simulated frontoparallel wall, which was moved in forward-backward direction at different frequencies $(0.05-0.5 \mathrm{~Hz})$. We covaried the amplitude of the movement with frequency in order to keep the wall velocity constant. We kept the amplitudes small (and thereby visual motion roughly at detection threshold) because we believe these amplitudes to be relevant for posture. This should be compared with Lestienne et al. (1977) and van Asten et al. (1988a) who generally employed larger amplitudes, which are more relevant for walking or running. We found that in an intermediate frequency regime, the action-perception cycle displays the typical phase-locked characteristics observed earlier (van Asten et al. 1988a). However, outside this regime coherence sometimes breaks down, and other types of coordination between postural sway and visual motion were observed. We observed both relative coordination, where there is a clear preferred phase relationship without stimulus and response being phase-locked, and absence of any coordination. These phenomena can be explained by an instability of the underlying phase dynamics, and observing it represents clear evidence for an effect of visual motion on the temporal structure of postural sway in the absence of phaselocking. A stable linear system that is passively driven by an additive force cannot display relative coordination (because the amplitude goes to zero when the system approaches the instability).

The paper is structured as follows. In the next section we provide a survey of the relevant theoretical backdrop by reviewing the linear dynamic model of postural control in a moving visual field and by presenting a discrete time dynamical model for the relative phase between sway and visual motion. In the Methods section we describe the experimental set-up employed, the nature of the measures taken, and we describe how the dynamical model is fitted to experimental data. In the Results section we present examples from the data illustrating the rich dynamics, and we report quantitatively the main results at the level of relative phase. Our conclusions are detailed in the Discussion section.

\section{Dynamic models of the action-perception cycle}

\subsection{The linear oscillator model for postural control in a moving visual environment}

As a point of reference, we review the model of Schöner (1991). It is based on the following assumptions. (1) The state of the postural control system can be described by the position, $x$, of the eye in the inertial frame, measured in forward-backward direction. (2) For a resting visual surround, the posture control system generates a stable fixed point of this variable at $x=0$ by choice of coordinates. (3) Visual information couples into this dynamics through the expansion rate, $e(x, t)$, of the visual surround. Mathematically,

$\ddot{x}+\alpha \dot{x}+\omega_{0}^{2} x-\sqrt{Q_{x}} \xi_{t}=-c_{\text {env }} e(x, t)$

with damping coefficient $\alpha$, eigenfrequency $\omega_{0}$ and noise strength, $Q_{x}$, where $\xi_{t}$ is gaussian white noise (zero mean, unit variance). The expansion rate:

$e(x, t)=\frac{\dot{x}-\dot{D}(t)}{x-D(t)}$

couples with strength $c_{\text {env }}$ such as to stabilise posture where $D(t)$ is the movement of the visual surround. For a sinusoidally moving surround, $D(t)=D_{0}+$ $D_{r} \sin \left(\omega_{D} t\right)$, moving at a mean distance $D_{0}$ with an amplitude $D_{r}$ and frequency $\omega_{D}$, the postural response (asymptotic solution of the deterministic part):

$x(t)=r_{0} \sin \left(\omega_{D} t+\phi_{0}\right)$

is harmonic with the same frequency as the visual motion. Amplitude and relative phase are given by:

$$
\begin{aligned}
& r_{0}=\frac{c_{\mathrm{env}} \omega_{D} D_{r}}{\sqrt{\left(\omega_{0}^{2}-\omega_{D}^{2}\right)^{2} D_{0}^{2}+\left(\alpha D_{0}+c_{\mathrm{env}}\right)^{2} \omega_{D}^{2}}} \\
& \tan \phi_{0}=\frac{\omega_{0}^{2}-\omega_{D}^{2}}{\left(\alpha+c_{\mathrm{env}} / D_{0}\right) \omega_{D}}
\end{aligned}
$$

The dynamics of the action-perception cycle can be studied by looking at solutions of the form $x(t)=r(t) \sin \left(\omega_{D} t+\phi(t)\right)$ so that the relative phase, $\phi(t)$, 
expresses the temporal relationship between sway and visual motion. A dynamics of relative phase can be derived by transforming (1) into polar coordinates and applying the averaging method (Guckenheimer and Holmes 1983):

$\phi=A-B \sin \phi+\sqrt{Q_{\varphi}} \xi_{t}$

with $A=\left(\omega_{0}^{2}-\omega_{D}^{2}\right) / 2 \omega_{D}$ and $B=c_{\text {cnv }} D_{r} / 2 r_{0} D_{0}$. For the averaging method to apply, one has to assume that the changes in relative phase and in amplitude ( $\dot{\phi}$ and $\dot{r})$ are slow compared with the driving frequency. This is a reasonable assumption for weakly perturbed oscillators. The noise in the relative phase dynamics is modelled in an ad-hoc fashion by additive gaussian white noise of strength $Q_{\varphi}$. The stationary relative phase of (3) is a stable fixed point of the relative phase dynamics. If the driving frequency, $\omega_{D}$, is significantly detuned from the eigenfrequency, $\omega_{0}$, this attractor could become unstable. This instability occurs at $A / B=1$, but is never reached in the linear model for which $|A / B|<1$ holds. The reason for this is that as the frequency difference increases $(|A|$ increases), the amplitude, $r_{0}$, vanishes (and hence $|B|$ increases). Therefore, solutions with finite amplitude but unstable relative phase do not exist in this linear model.

In light of the outcome of our experiment, we note that a very similar equation for the relative phase can be derived by averaging for driven non-linear oscillator models (Guckenheimer and Holmes 1983, Sect. 4.2). In this case, the instability of the fixed point for relative phase can be reached, however, by detuning the driving frequency from the eigenfrequency, because the amplitude of the oscillator does not necessarily go to zero when the stability limit for relative phase is reached.

\subsection{The stochastic sine-circle map}

For quantitative comparison with our experimental data, we use a generalisation of the dynamics of relative phase (4) which covers both the case in which such dynamics arise from a passively driven system as well as the case in which such dynamics arise from a driven active (nonlinear) oscillator. Because we estimate relative phase at discrete points in time (e.g. at peaks or valleys of position), we use a discrete time dynamical model. The simplest model compatible with the circular nature of relative phase is the sine-circle map (Bohr et al. 1984):

$\phi_{n+1}=\phi_{n}+a+b \sin \left(\phi_{n}-\phi_{0}\right)+\sqrt{Q} \xi_{n}$

where $\phi_{n} \in S^{1}$ is the relative phase at time $n$, and $a, b$ and $\phi_{0}$ are parameters to be explained below. For comparison with noisy data, we have included a stochastic component through independent gaussian random forces $\xi_{n}$ (zero mean, unit variance) acting with strength $Q$.

Maps of this type arise through Poincaré sections in models of driven non-linear oscillators (Bohr et al. 1984). In these cases the parameter $a$ arises as the difference in frequency between the oscillator and the forcing function, and $b$ reflects the coupling strength of the forcing function and the oscillator. We employ the model in a phenomenological spirit by mapping the observed relative phase series onto solutions of this dynamical system. In this case the parameters arise from numerical fits to experimental data (see Methods). The meaning of the parameters is best interpreted in terms of how the fixed points and their stability depend on the parameters. To discuss this, we first consider the deterministic dynamics by setting $Q=0$. The parameter $\phi_{0}$ can be absorbed into a shift of coordinates and therefore does not affect the stability of the dynamics. In the Results section we show that $\phi_{0}$ can be approximated as zero. Therefore, we will set $\phi_{0}=0$ for the purposes of this discussion.

The circle map can be visualised by the return map, a plot of $\phi_{n+1}$ versus $\phi_{n}$. In Fig. 1 we show examples for different values of the parameters $a$ and $b$. Note that when $|a|<|b|$ the system has two fixed points, only one of which is stable (which one depends on the sign of $b$ ). The stable fixed point is the one for which the derivative of the right-hand side of (5) is smaller than 1 in absolute value. More precisely, for $|a|<|b|$ the two fixed points are:

$\phi_{f}^{1}=-\arcsin (a / b)$

$\phi_{f}^{2}=\pi+\arcsin (a / b)$

Stability of the fixed points is calculated by linearising the map in the neighbourhood of the fixed points. Take $\phi_{n}=\phi_{f}^{1}+\delta_{n}$ and $\phi_{n+1}=\phi_{f}^{1}+\delta_{n+1}$ with $\delta_{n}$ and $\delta_{n+1}$ small perturbations. By substitution in (5) and expanding the sine function up to the second order, we obtain:

$\delta_{n+1}=\left(1+b \sqrt{1-(a / b)^{2}}\right) \delta_{n}$

For $b<0$ we find for an initial perturbation $\delta_{0}$ :

$\delta_{n}=\delta_{0}\left(1-\sqrt{b^{2}-a^{2}}\right)^{n}$

which converges to zero if $|1-\lambda|<1$, in which case $\phi_{f}^{1}$ is stable. Here we have defined the local stability parameter $\lambda=\sqrt{b^{2}-a^{2}}$. The other fixed point is unstable with $\delta_{n}=\delta_{0}(1+\lambda)^{n}$ diverging. For $b>0$, the roles of the fixed points $\phi_{f}^{1}$ and $\phi_{f}^{2}$ are reversed. Intuitively, large $b$, or in terms of underlying oscillators, strong coupling, implies large $\lambda$ inducing stable phase-locking. Stability is similarly enhanced by small $a$, i.e. good match of driving frequency and eigenfrequency. Equation (8) describes the decay of perturbations of relative phase in units of discrete time. Later in this article we compare relaxation processes across frequencies. Because the discrete time
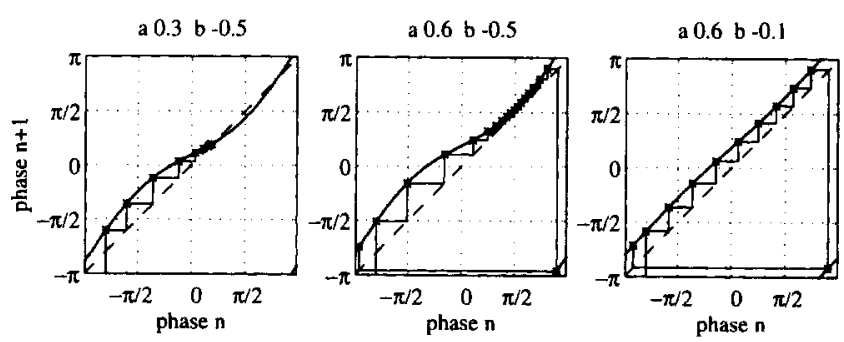

Fig. 1. Examples of return maps of the sine-circle map for several parameter values. Note that phase is a periodic variable, i.e. phases moving off at the top reappear at the bottom and phases moving off at the left reappear at the right. Left panel, absolute coordination; middle panel, relative coordination, right panel, no coordination 
units are determined by the cycle time, this implies that the size of the relevant time units for measuring the relaxation process varies with frequency. To make comparisons across frequency, we rescale the local stability as $\bar{\lambda}=\lambda / \delta t$ where $\delta t$ is the relevant time unit (quarter of a cycle). This rescaling converts the relaxation process to units of absolute time. For small $\delta t,(8)$ may be approximated as:

$\delta_{n}=\delta_{0} \exp \left(-T / \tau_{\mathrm{rel}}\right)$

with $\tau_{\text {rel }}=1 / \tilde{\lambda}$ the relaxation time and $T=n \delta t$ the time at which $n / 4$ cycles have run past.

At $|a|=|b|$ the model exhibits a tangent bifurcation: the two fixed points collide at phase $\pm \pi / 2$. When $|a|>|b|$, no fixed points exist. Note that when $|a|$ is slightly larger than $|b|$ (see Fig. 1), a narrow tunnel exists where the return map comes close to the diagonal. In this tunnel the relative phase takes small steps, whereas it takes large steps outside the tunnel. This means that the oscillator is not phase-locked to the forcing function but is still influenced by it so that the oscillator lingers for longer periods of time near a relative phase of $\pm \pi / 2$. When $|a|$ is much larger than $|b|$ (see Fig. 1), the phase takes constant steps without any significant slowing down. This means that the oscillator is moving independently of the forcing function. We may define a global stability parameter, $\gamma$, which indicates how close the system is to one of the bifurcations: $\gamma=a /|b|$ (which one depends on the sign of $\gamma$ ). For $|\gamma|<1$ one stable and one unstable fixed point exist; for $|\gamma|>1$, no fixed point exists. This parameter characterises the dynamics overall, also where the local stability parameter does not exist.

Three types of coordination frequently observed in rhythmic activity can be mapped onto the three regimes illustrated in Fig. 1. This classification of type of coordination goes back to von Holst (1937/1973). Absolute coordination in which relative phase is maintained within a narrow interval can be mapped onto the regime with a stable fixed point of relative phase corresponding to phase-locked behaviour. Relative coordination, in which all relative phase values occur but with unequal probability so that there is a preferred relative phase, can be mapped onto the regime just following the tangent bifurcation. The preferred relative phases are the points in the tunnel, where the return map comes close to the diagonal. Finally, no coordination, in which there is no regularity in the relative timing of two rhythmic signals, can be mapped onto the regime in which $|a|$ is much larger than $|b|$. Based on experimental data and fitted return maps, we shall discuss this mapping in more detail in the Results section.

The model of (5) has a very rich dynamics, not all of which is relevant to our system. The model allows for non-1-1 locking, which we never observed. Further, when $\lambda>2$, the stable fixed point becomes unstable again. Finally, when $|b|>1$, the map becomes non-invertible, and chaos may occur (Jensen et al. 1984). We have neither observed these two phenomena nor obtained reconstructions of return maps corresponding to these parameter regimes, so we will not discuss these cases.
The discrete time dynamics of (5) can be related to continuous time dynamics of relative phase as discussed in the previous section. Clearly, in view of the richness of the discrete dynamics much exceeding that of a firstorder differential equation, this relationship is not exact. However, in the limit of the time unit, $\delta t$, of the discrete dynamics becoming infinitesimally small, $\left(\phi_{n+1}-\phi_{n}\right) / \delta t$ approximates $\dot{\phi}$. In this limit (5) becomes:

$\dot{\phi}=\tilde{a}+\tilde{b} \sin \left(\phi(t)-\phi_{0}\right)+\sqrt{\tilde{Q}} \xi_{t}$

where the parameters have been rescaled according to $\tilde{a}=a / \delta t, \tilde{b}=b / \delta t, \tilde{Q}=Q / \delta t$ and $\xi_{t}$ is gaussian white noise of unit variance. In our experiments, relative phase is obtained at four points in each cycle, so $\delta t$ equals a quarter cycle time. Even though this is not infinitesimal, we shall employ these rescaled parameters to compare relative phase dynamics across frequencies.

Adding noise to the sine-circle map does not change the dynamics very much (unpublished observations; for low noise levels see Wiesenfeld and Satija 1987). When there is a stable fixed point, the noise causes the phase values to scatter around it. The width of the distribution of phase values, when the noise is not too large, can be characterised by the standard deviation of relative phase and is given by (Schöner 1991):

$S D_{\varphi}=\sqrt{Q / \lambda}$

Further, the bifurcation is softened by the noise: for $|b|$ slightly larger than $|a|$, the fixed point has low stability, and due to the noise the system can escape easily from the attractor and wrap around. The stationary distribution cannot be calculated analytically. It can be solved numerically though by discretisation of the transition matrix and by calculation of the left and right eigenvectors with eigenvalue 1 (Reichl 1980).

\section{Methods}

\subsection{Experimental set-up}

Red/green stereograms were generated by a SUN4 workstation and were projected onto a translucent screen $(2.5$ by $2 \mathrm{~m}$ ) by a video projector. The subject wore a pair of goggles that contained red/green filters and limited the field of view to approximately $120 \mathrm{deg}$ wide by $100 \mathrm{deg}$ high. Because of the restriction of the viewing range, the edge of the screen was not visible to the subject. The subject stood approximately $50 \mathrm{~cm}$ in front of the screen wearing a helmet on which six infrared light-emitting diodes were mounted. The positions of these diodes were measured with two cameras of a Watsmart system (Northern Digital Inc).

The two-dimensional (2D) coordinates of the two cameras were converted real-time into $3 \mathrm{D}$ coordinates and sent to the workstation. This computer was programmed to generate a new stereogram of the wall from the current viewpoint of each eye of the observer. With this set-up, every frame $(15 \mathrm{~ms})$ provided a new view of the simulated wall. The mean $3 \mathrm{D}$ position of the 
view points and the orientation of the head were stored together with the position of the stimulus for later analysis. These signals were sampled at a rate of $66 \mathrm{~Hz}$. The details of the feedback loop are explained in Dijkstra et al. (1994) but are not so important here as the physical distance from subject to screen was equal to the suggested distance. It should be noted that the orientation of the eyes in their sockets was not measured.

\subsection{Stimuli}

The stimuli simulated a fronto-parallel wall covered with 140 stereo dots, each with a size of $0.2 \mathrm{deg}$ by $0.2 \mathrm{deg}$. The density of dots was uniform per solid angle as seen from the position at which the experiment started. The dots lay in an annulus between 10 deg and 45 deg visual eccentricity. The hole in the middle of the stimulus was made to suppress the visibility of aliasing effects, which were most visible in the foveal region. The spatial resolution of the system was 1152 by 900 pixels, and the frame rate was $66 \mathrm{~Hz}$.

The wall was suggested at a mean distance of $50 \mathrm{~cm}$. Distance was suggested both by stereo vision as well as by simulation of the appropriate displacement of the image on the screen, using feedback of the eye position of the observer. Stereo vision is not essential but makes prolonged viewing more comfortable. The wall was sinusoidally moved in the forward-backward direction with frequencies of $0.05,0.1,0.2,0.3,0.4$ and $0.5 \mathrm{~Hz}$. The amplitude was covaried with frequency so as to keep the mean absolute velocity of the wall constant. In this way we kept the perceptual strength roughly constant: all stimuli were near the threshold where stimulus motion could be consciously perceived. We took amplitudes of $10,5,2.5,1.67,1.25$ and $1 \mathrm{~cm}$, respectively. All conditions except for $0.05 \mathrm{~Hz}$ had two perturbations of phase of $180 \mathrm{deg}$ at the point of maximum velocity (so there was no discontinuity in position, see Fig. 2, upper panel). The perturbations occurred at random moments in time but were separated by at least $35 \mathrm{~s}$, thus ensuring that there were enough unperturbed cycles to estimate the relaxation time. Because of the small number of cycles in the $0.05 \mathrm{~Hz}$ condition, we had only one perturbation.

Each condition was repeated six times, and the order of trials was balanced in a latin square design. With this design every condition followed every other condition precisely once. The experiment was conducted in two sessions of approximately $1 \mathrm{~h}$ each and always started with two trials at $0.2 \mathrm{~Hz}$ for adaptation of the subject. In all, there were 40 trials for each subject. Each trial lasted for $140 \mathrm{~s}$, and the first $20 \mathrm{~s}$ were used for adaptation and were not stored.

\subsection{Data analysis}

Data analysis was done in two ways: a linear time invariant analysis and a dynamic approach based on the time series of relative phase, as described in Schöner and Kelso (1988). The data for both types of analysis were the sinusoidal motion of the wall and the postural response of the subject in fore/after direction sampled at $66 \mathrm{~Hz}$ for $2 \mathrm{~min}$.
3.3.1 Linear systems theory. The linear time-invariant analysis comprised calculating Fourier transforms of the drive and the response of the subjects from which we determined the magnitude squared coherence (MSC), the phase and the gain at the driving frequency. All spectra were calculated with a Welch procedure (Marple 1987) in order to obtain consistent estimates. From each trial we excluded an 8-s period after each perturbation to assure stationarity, and for each of the remaining three parts we used 7 segments and a factor 3 zero padding. The MSC depends strongly, the phase and gain very weakly on the number of segments (Carter 1987).

3.3.2 Time series of relative phase. The dynamic analysis involved calculating a discrete time series of relative phase (phase of response minus phase of stimulus; Kelso et al. 1990). This discrete estimate of relative phase has the advantage over continuous estimates that the signals need not be sinusoidal. From the input and response data the significant extremes both of position and velocity traces were picked using a peakpicker. Before peakpicking, the data were smoothed using a gaussian window with a standard deviation of $0.18 \mathrm{~s}$ (for the $0.05 \mathrm{~Hz}$ condition this was $0.64 \mathrm{~s}$, and for $0.1 \mathrm{~Hz}$ this was $0.36 \mathrm{~s}$ ). The criterion for significance of an extremum was a fraction of the range (the difference between maximum and minimum) in a segment of a cycle before and a cycle after the extremum. An extremum (e.g. a peak) in position was accepted as significant when it differed by more than $20 \%$ of the range from the neighbouring extrema (e.g. two valleys). For an extremum in velocity, the criterion was $50 \%$. We chose these percentages differently because velocity tended to be more peaked than position. The results depend weakly on these percentages. From the 8 time series of extrema (maxima and minima, both position and velocity of both stimulus and response), relative phase was calculated with the stimulus as reference and the response as target. This was done by matching each extremum in the reference signal to all extrema in the target signal of the same type and within half a cycle before and half a cycle after the extremum. A relative phase value was calculated by taking the time difference between two extrema of the same type and dividing this by the time difference between two extrema in the reference signal (this is the cycle time of the drive). To this phase value we added $2 \pi$ times the number of wraps, i.e. the difference in number of cycles between reference and target. When there is no extremum in the target signal, the number of wraps is decreased by 1 . When there is more than one extremum in the target signal, the number of wraps is increased by 1 for each extremum except the first. As the time value of this relative phase in the time series we used the time of the target extremum. The result of these manipulations is four time series of relative phase: maxima and minima of position and of velocity. Because these four time series were not very different, we combined them in one overall time series of relative phase.

3.3.3 Measures calculated from the time series of relative phase. We determined mean phase and angular 
deviation from the time series of relative phase, using circular statistics (Batschelet 1981):

$r \exp (i \bar{\phi})=\sum_{n} \exp \left(i \phi_{n}\right)$

with $n$ running over all phase values. The mean phase is $\bar{\phi}$, and the angular deviation is $s=\sqrt{2(1-r)}$. Mean phase is a measure for the time delay between input and response, and angular deviation is a measure for the stability of the response. It should be noted that angular deviation is biased: it tends to be underestimated for small samples (Batschelet 1981, p 46). In our experiment the number of phase values depends on the mean frequency of the response and can vary by a factor 10 . There are correction procedures for this bias (which assume specific distributions), but we will present the angular deviation as calculated above and not make strong conclusions from it alone. The advantage of the angular deviation over most of the stability measures presented below is that it can be calculated for all trials, no matter how unstable. We excluded the phase values in a period of $8 \mathrm{~s}$ after the perturbation, because mean phase and angular deviation are measures for the stationary behaviour.

As a further measure of the stability, we calculated the winding number defined as $W=\left(\phi_{N}-\phi_{0}\right) /(2 \pi N)$, with $N$ the number of phase values. It measures the mean wrapping of the response relative to the drive. Negative winding number means that the response is slower than the drive (this occurs for high driving frequencies), and a positive one means that the drive is slower than the response (this occurs for low driving frequencies). A winding number near zero means that the response is phase-locked to the drive.

3.3.4 Relaxation time. The relaxation time, the time it takes the system to regain its phase-locked behaviour after a perturbation, was estimated as follows: we defined a band around the local mean of relative phase of 1.5 times the local angular deviation. Local mean phase and angular deviation were calculated from a segment starting $8 \mathrm{~s}$ after the perturbation and with a length of four cycles. After a perturbation the phase generally leaves this band. We calculated re-entry time as the time between the perturbation and the time of re-entry into the band. From this we calculated the relaxation time by fitting an exponential to the points outside the band and the first six points inside the band (cf. Fig. 2, middle panel). The fit was implemented by a non-linear Levenberg-Marquardt routine (Press et al. 1988). The points inside the band were given a larger weight because they were less prone to noise. We did not use linear regression on the logarithm of the phase values because the phase values are not necessarily all at the same side of the local mean phase. If the relative phase did not leave the band or if many wrappings occurred near the perturbation (cf. Fig. 4, middle panel), we excluded the perturbation. The relaxation time depends somewhat on the parameters used, but the trend with frequency does not. We calculated the relaxation time of a trial by averaging the relaxation times of the two perturbations within a trial.
3.3.5 Fitting of the return map and histogram. We estimated directly the parameters $a, b$ and $Q$ of the stochastic sine-circle map. We did this both by fitting the return map and by fitting the histogram. The return map fit was a linear least squares fit of $y=a+b x$ with $y=\phi_{n+1}-\phi_{n}$ and $x=\sin \phi_{n}$, where $\phi_{n}$ is the $n$th relative phase. The unexplained variance of the fit gives an estimate of the noise strength $Q$. We included the phase values directly after a perturbation in the fit because these provide information about the relaxation towards the attractor. Further, we excluded $2.5 \%$ of the phase values from the return map fits. This was done because some of the phase values were outliers. Some of these outliers in the return map were caused by small (but fast) bumps in the response data, leading to an extra pair of extrema in the velocity and thus to a wrap in the time series of relative phase. Especially for the phase-locked trials, where all phases scatter around the attractor, these outliers changed the fitted parameters considerably. Excluding the outliers from the return map fit also led to better agreement of the parameters from the fit of the return map and of the histogram. As a measure of significance of the fit, we used its $F$ value. We excluded the results of a fit when the corresponding significance level exceeded $10 \%$.

The histogram was constructed from the time series by grouping the phase values in bins. We calculated the stationary distribution of the stochastic sine-circle map (Reichl 1980) and used a non-linear least squares fit procedure (Levenberg-Marquardt) to fit the parameters of the stationary distribution to the experimentally observed histogram. The starting values of these fits were the parameters from the return map fit. When we found an attractor $(|a|<|b|)$ with the return map fit, we fitted $a$ and $Q$, otherwise we fitted $a$ and $b$. Fitting all three parameters led to unstable results, because the histogram has only two conspicuous features: a mean and a width. We fitted $a$ and $Q$ when we found an attractor with the return map fit, because $b$ was fitted very accurately from the return map (it is mainly determined by the slope at the fixed point). We fitted $a$ and $b$ when we did not find an attractor with the return map fit because we wanted the stability measure $\gamma$ to be determined as accurately as possible. The number of bins was adapted to the number of phase values and the angular deviation of the phase values. The results of the fit depend weakly on the precise number of bins. As a measure of significance of the fit, we took the $\chi^{2}$ value, which we used to test whether the histogram differed significantly from a uniform distribution. We excluded the results of a fit when the histogram did not differ significantly $(p>1 \%)$ from a uniform distribution.

For comparison of the parameters $a, b$ and $Q$ with their counterparts in the continuous model (4), we rescaled the parameters with the sampling frequency of the time series of relative phase (four times the mean frequency of response).

\subsection{Subjects}

Six subjects with normal or corrected to normal vision were tested under all conditions. Three of the subjects 

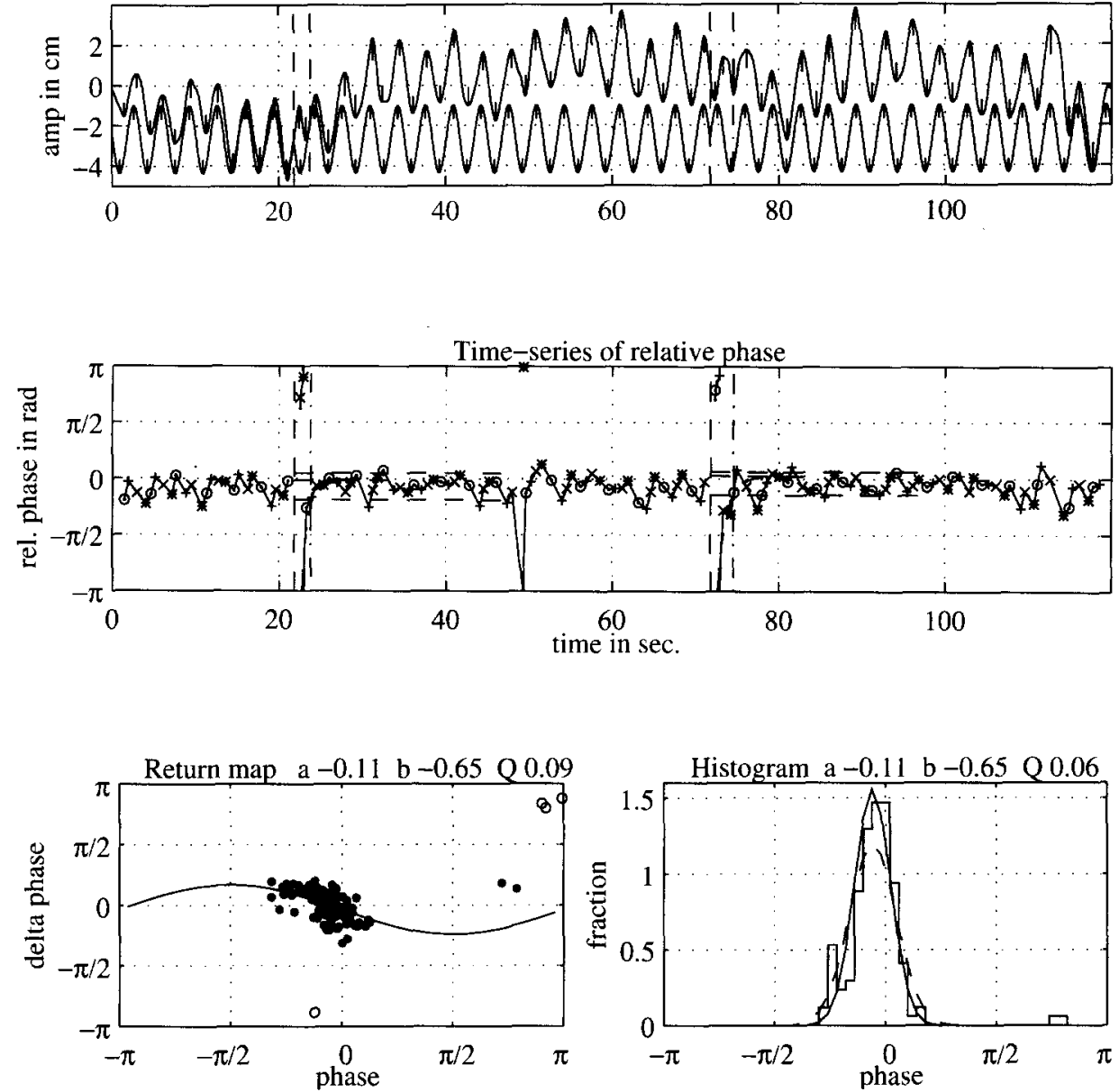

Fig. 2. Example of a data record at medium driving frequency $(0.3 \mathrm{~Hz})$. Upper panel, position traces of visual drive (shifted down for visibility) and postural response. The position of the short solid iertical lines denotes the extrema as picked by the peakpicker, their length denotes the criterion used in picking. The dashed vertical line denotes the time of perturbation and the dash-dot line, the re-entry time. Positive means movement towards the wall (standing more on your toes), negative means away from the wall. Middle panel, derived time series of relative phase (solid line) with exponential fit of the relaxation (dashed line). Symbols denote the different types of phase values: $x$ denotes peaks position, $o$ denotes valleys position, + denotes peak velocity, and $*$ denotes valley velocity. Also indicated are the time of the perturbation (dashed vertical line) and the re-entry time (dash-dot vertical line). The middle dashed horizontal line after a perturbation denotes the zero level of the relaxation. The two outer dashed horizontal lines are plus and minus 1.5 times the angular deviation around the zero level. Lower left panel, derived difference return map with scatterplot of the data (open dots are excluded from the fit) and the curve of the best fit. The parameters of the fit are indicated. Lower right panel, derived histogram of relative phase (staircase plot) with the best fit (solid line) and the stationary distribution as calculated with the parameters of return map (dashed line) were familiar with the purpose of the experiment. It should be noted that some of the results (especially relative coordination) were not expected at the time of the experiment. Four subjects had participated in a previous study (Dijkstra et al. 1994). Subjects were instructed to look at the centre of the stimulus and to stand relaxed. Subjects stood on a firm stable support in normal Romberg posture.

\section{Results}

Generally the subjects responded to the sinusoidally moving wall with a sinusoidal postural response in the fore/aft direction. They displayed a rich set of dynamical behaviour: tightly phase-locked (absolute coordination) in the middle frequency range, both absolute coordination and no coordination at low frequencies, and all three coordination patterns at high frequencies. We used a rough classification based on the global stability parameter $\gamma$ to obtain an idea of the frequency of occurrence of the various coordination patterns. When $|\gamma|<0.75$, we classified a trial as absolutely coordinated; when $|\gamma|$ was between 0.75 and 1.25 , we classified a trial as relatively coordinated; and when $|\gamma|>1.25$, we classified it as uncoordinated. Proceeding in this way we found $81 \%$ of the trials to be absolutely coordinated, $11 \%$ to be relatively coordinated and $8 \%$ to be uncoordinated. The amplitude of sway reflected the amplitude of visual motion at all frequencies: the gain was generally independent of frequency and was close to 1 for most subjects. A more detailed analysis of the spatiotemporal characteristics of sway will be reported elsewhere, as it exceeds the scope of this paper. To give the reader an idea of the rich behaviour, we discuss a few data sets.

\subsection{Examples of dynamic behaviour}

In Fig. 2 we present an example of absolute coordination at a mid-range driving frequency. In the upper panel we show the trajectories of the stimulus and the response of the subject. The subject is in-phase with the stimulus and is phase-locked. In the middle panel we show the corresponding time series of relative phase. The relative phase scatters around zero. The lower left panel shows the return map of relative phase in a slightly different format, which is handy for fitting the parameters:

$\phi_{n+1}-\phi_{n}=a+b \sin \left(\phi_{n}\right)$

The dots are data points, while the line represents the above function based on fitted parameter values (only the 

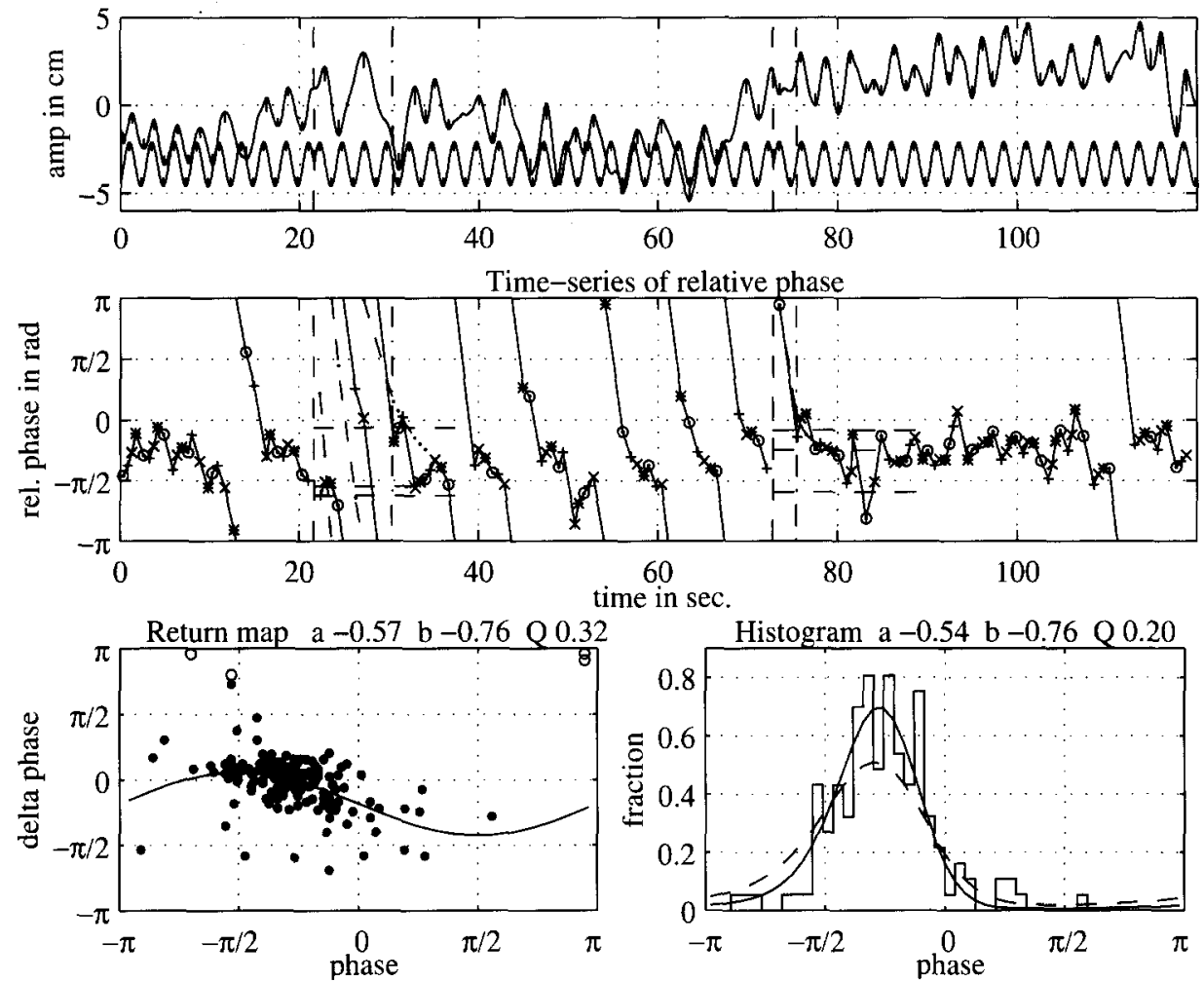

Fig. 3. Example of a data record at high driving frequency $(0.4 \mathrm{~Hz})$. This trial shows relative coordination. For details, see legend of Fig. 2 solid dots are included in the fit, the open dots are excluded, see the Method section). The existence of a stable fixed point is clear from the negative slope of the fitted return map at the intersection with the $\phi$ axis, reflected also by the spread of the data points. This is corroborated in the lower right panel, where we show the histogram of relative phase indicating a clear peak centred on the mean phase. Note that the predicted distribution from the fit of the return map (dashed line) is close to the optimal fit of the histogram (solid line), just as in the following figures.

In Fig. 3 we present an example of relative coordination at a high driving frequency. In the middle panel in the stretch between the two perturbations one sees slow dynamics at a phase of roughly $-\pi / 2$ and fast dynamics at the other phases (cf. Fig. 1). The trajectories show that postural sway is still oscillatory in spite of the loss of phase locking. This indicates that sway motion is actively generated and not passively driven by visual motion. Because the stretches before the first perturbation and after the second are phase-locked, one does not see the relative coordination clearly reflected in the return map and the histogram. However, fitting the return map and the histogram only for the phase values between the two perturbations, we found $a=-0.53$ and $b=-0.54$. It is interesting to note that the perturbations seem to cause a switch from one type of dynamics to another. Also interesting is the finding that the two relaxation times are very different: the first is $4.0 \mathrm{~s}$ and the second, $1.4 \mathrm{~s}$. This is consistent with the finding that the stretch after the first perturbation has a low local stability, whereas the stretch after the second has a high local stability.
In Fig. 4 we present an example of the absence of coordination at a high driving frequency. In the middle panel one can see that the subject continuously wraps in phase, indicating that he oscillates at his own frequency, different from the driving frequency. Like relative coordination, this form of uncoordinated sway is indicative of active generation of sway motion. The relative phase is very variable, as can be seen from the return map, where the data points fill the lower part (with delta phase negative). The noise strength $Q$ is very large. Note that the histogram is not a very useful tool in this case because it does not differ significantly from a uniform distribution.

\subsection{Mean relative phase, stability and mean response frequency}

In this section we present the main findings. We tested all effects with a one-way ANOVA with independent factor frequency for each subject separately at a significance level of $5 \%$. In general, most effects of frequency presented here were significant.

In Fig. 5 we plotted the mean phase difference between visual drive and postural response as calculated from the time series of relative phase. All subjects had a significant frequency effect for mean phase. From the figure we observe that subjects are advanced relative to the stimulus at frequencies below $0.2 \mathrm{~Hz}$, are almost in-phase near $0.2 \mathrm{~Hz}$ and are delayed at high frequencies. This frequency dependence is predicted from (3) and Fig. 3 of Schöner (1991), in terms of which the mean eigenfrequency of the postural system is in all subjects 

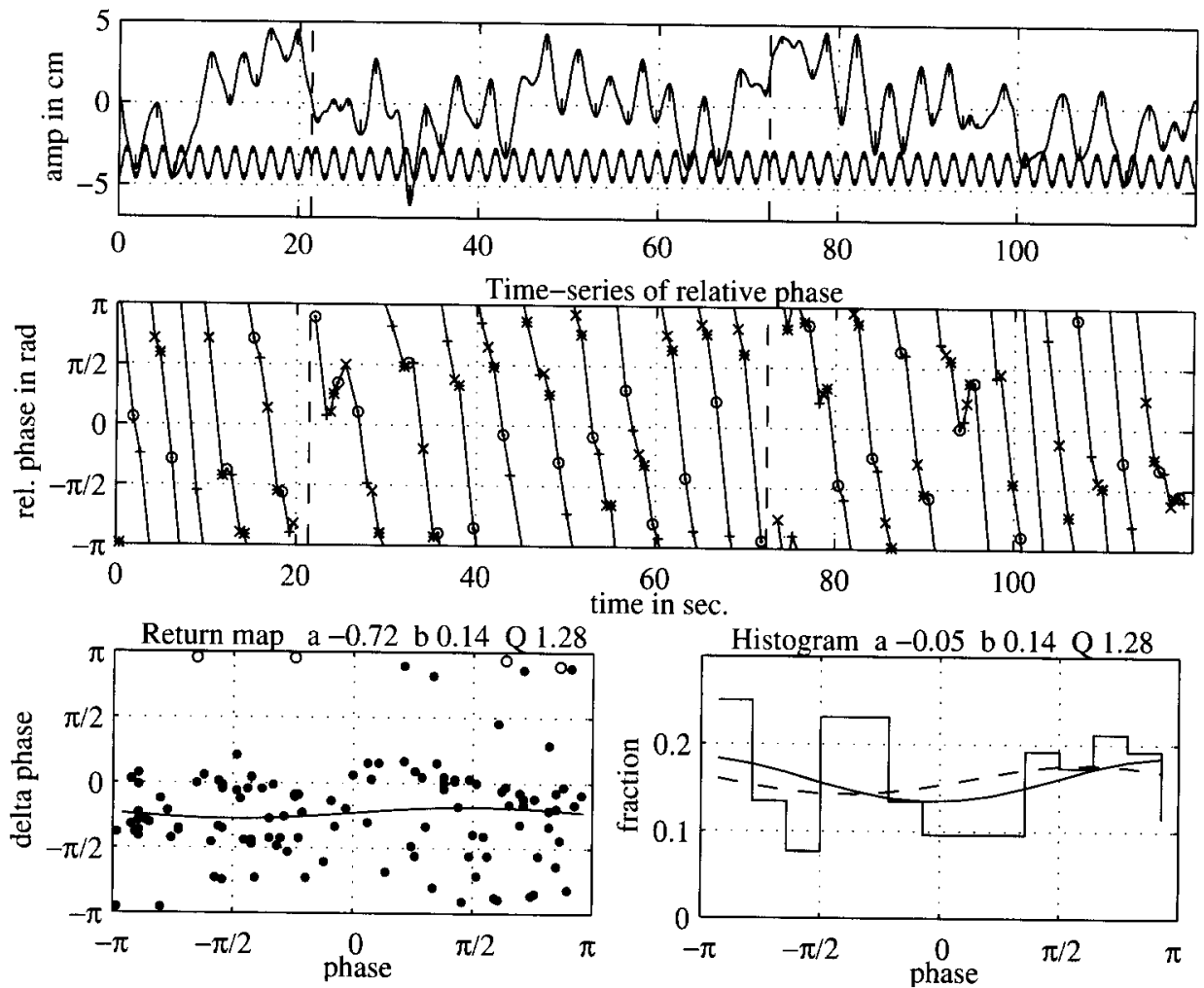

Fig. 4. Example of a data record at high driving frequency $(0.5 \mathrm{~Hz})$. This trial shows no coordination. For details, see legend of Fig. 2

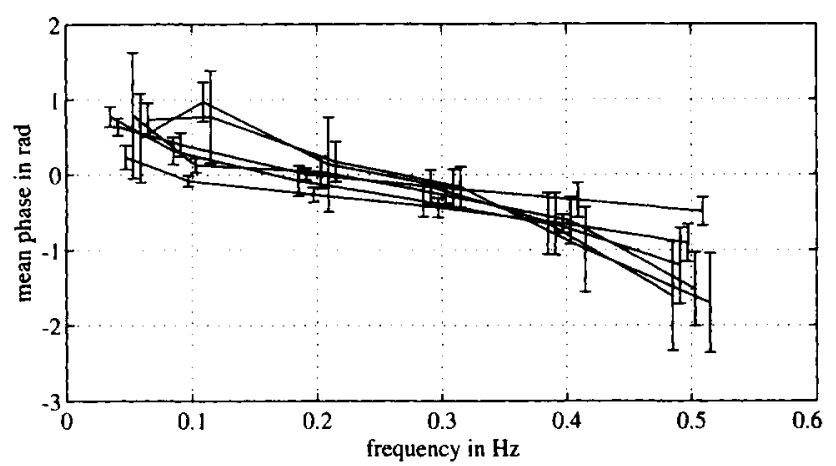

Fig. 5. Mean phase as a function of driving frequency for all subjects. The curves for different subjects are horizontally displaced for better viewing. Error bars denote standard deviation

approximately $0.2 \mathrm{~Hz}$. Also note that there is a tendency for the error bars to be larger for the low and high frequencies. This enhanced variability across trials reflects the reduced temporal stability at these frequencies (see below). The mean phase as calculated from the time series of relative phase correlates significantly with the mean delay as calculated from the spectrum: the correlations for each subject are in the range 0.62 to 0.82 , mean 0.76 . The mean phase at a driving frequency of $0.2 \mathrm{~Hz}$ for the four subjects who also participated in a previous study (Dijkstra et al. 1994) matches very closely the results in that study for equivalent conditions (distance of $50 \mathrm{~cm}$ ): subject $\mathrm{CG}$ has a mean phase near $0.27 \mathrm{rad}$ in both experiments, the other subjects have a mean phase close to zero in both experiments.
In Fig. 6 we plotted the angular deviation. This measure for the temporal stability of the action-perception coupling has a significant frequency effect for all but two subjects. The figure shows the stability to be higher (variability lower) in the range $0.2-0.3 \mathrm{~Hz}$ with a decrease in stability towards the low and high frequencies. The two subjects lacking significance can be seen to have higher variability throughout, which also increases towards the higher and lower frequencies. The linear dynamical model does not predict any effect of frequency on stability (cf. (15) of Schöner 1991) so that the observed effect is evidence for non-linearities. Because of the bias of the angular deviation (see Methods), we cannot directly compare the stability of the low and high frequencies. The angular deviation correlates significantly with the magnitude squared coherence (MSC) calculated from the spectrum: the correlations for each subject are in the range -0.66 to -0.87 , mean -0.78 . The correlations are negative because small angular deviation and high MSC both signify high stability.

In Fig. 7 we plotted the relaxation time. There was no significant effect of frequency on relaxation time for all but one of the subjects. Excluding the relaxations at the low frequency of $0.05 \mathrm{~Hz}$, where the sampling of the phase is problematic, the relaxation time is at a constant level between 2 and $3 \mathrm{~s}$, except for some outliers. For the low and high frequencies many perturbations had to be excluded because the subject showed no clear locking after the perturbation. Subjects did not differ in relaxation time but did differ in the number of included relaxations (ranging from $97 \%$ to $45 \%$, mean $72 \%$ ), especially at the low and high frequencies. These 


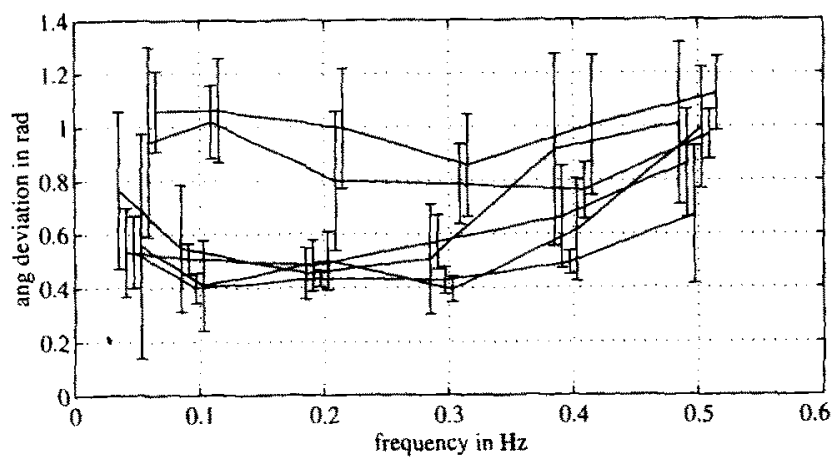

Fig. 6. Angular deviation of relative phase as a function of driving frequency for all subjects. The curves for different subjects are horizontally displaced for better viewing. Error bars denote standard deviation

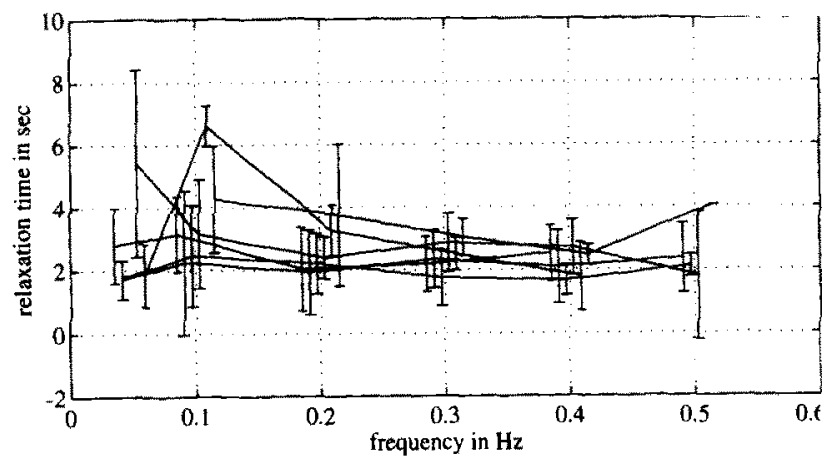

Fig. 7. Relaxation time as a function of driving frequency for all subjects. The curves for different subjects are horizontally displaced for better viewing. Error bars denote standard deviation

exclusions may lead to an underestimation of relaxation time at the low and high frequencies and therefore obscure an effect of frequency on relaxation time. In a previous study (Dijkstra et al. 1994) we reported a significant correlation between angular variance and relaxation time. Here we found correlations ranging from 0.05 to 0.62 , mean 0.38 . Half of these were significant at a level of $5 \%$, and all of them were lower than in the previous experiment. We cannot expect a strong correlation because our frequency manipulation does not induce a significant effect in the relaxation time and because the angular deviation is biased.

In Fig. 8 we plotted the difference between the mean frequency of the response and the driving frequency. The mean response frequency was calculated from the time series of relative phase. All subjects display a significant effect in this variable. For frequencies in the range $0.1-0.2 \mathrm{~Hz}$, we note that the mean response frequency equals the frequency of the input, except for two subjects. These subjects also showed a higher angular deviation at these frequencies. For other frequencies, especially at $0.5 \mathrm{~Hz}$, there are marked differences. Subjects have a tendency to respond with a higher frequency for the low driving frequency of $0.05 \mathrm{~Hz}$ and to respond with a lower frequency for the high driving frequencies. This type of behaviour cannot be exhibited by a purely linear system because for a linear system, the frequency of the response always equals the frequency of the input. Also note that

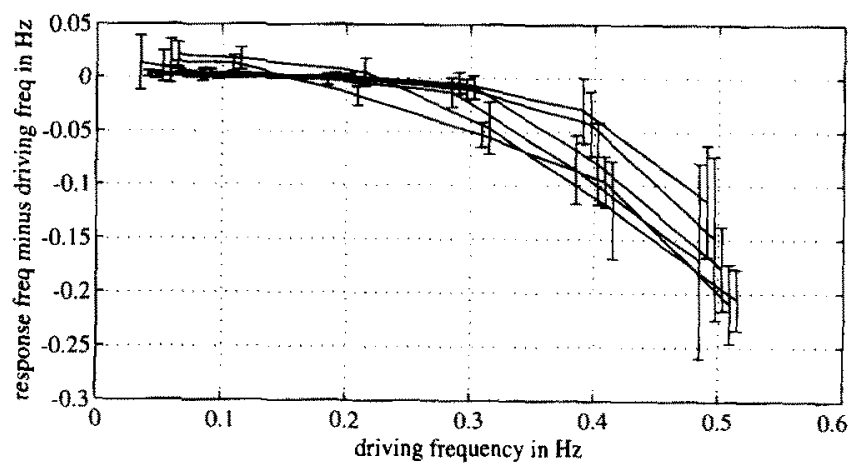

Fig. 8. Difference between mean frequency of response and driving frequency as a function of driving frequency for all subjects. The curves for different subjects are horizontally displaced for better viewing. Error bars denote standard deviation

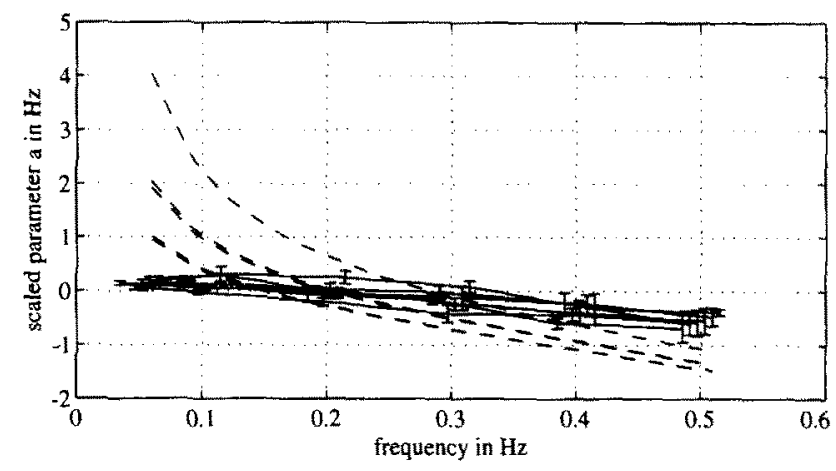

Fig. 9. Parameter $\tilde{a}$ of return map fit as a function of driving frequency for all subjects. The curves for difierent subjects are horizontally displaced for better viewing. Error bars denote standard deviation. Broken lines denote the best fit of the data using the first term on the right-hand side of (4)

the variability across trials as denoted by the size of the error bars is higher at the extreme frequencies, especially $0.5 \mathrm{~Hz}$, than at the middle frequencies.

\subsection{Relative phase dynamics}

In Fig. 9 we plotted the scaled parameter $\tilde{a}$ as calculated by fitting of the return map. All subjects have a significant frequency effect for this parameter. In Fig. 9 we also plotted (broken line) least squares fit using the first term on the right-hand side of (4). The fit resulted in an eigenfrequency (mean over all subjects) of $0.2 \mathrm{~Hz}$, individual eigenfrequencies ranging from 0.15 to $0.28 \mathrm{~Hz}$. However, the poor quality of the fit indicates deviations from the linear model which can be interpreted in terms of adaptation of eigenfrequency (this point will be elaborated in a subsequent paper).

In Fig. 10 we plotted the scaled parameter $b$. All subjects except one have a significant frequency effect for this parameter. The parameter $b$ can be interpreted as an indicator of the coupling strength between stimulus and response. The results indicate that the coupling strength is low in absolute value for $0.05 \mathrm{~Hz}$ and $0.1 \mathrm{~Hz}$ and higher for the range $0.2-0.5 \mathrm{~Hz}$. There is considerable variation between subjects for this measure. 


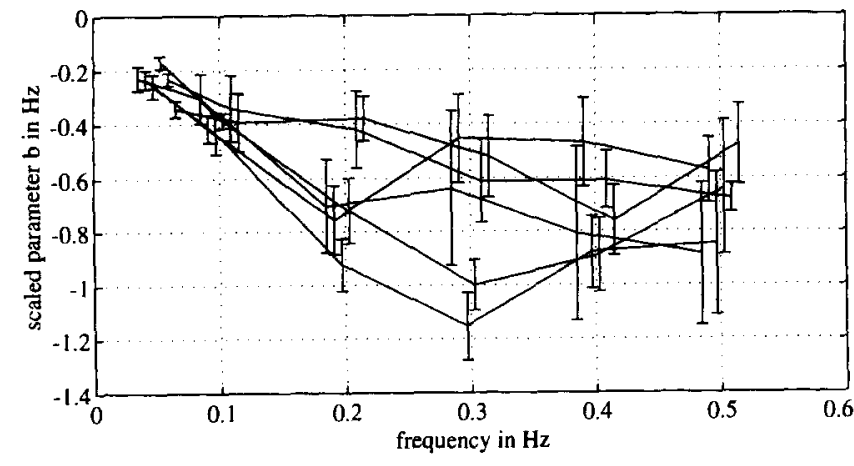

Fig. 10. Parameter $\tilde{b}$ of return map fit as a function of driving frequency for all subjects. The curves for different subjects are horizontally displaced for better viewing. Error bars denote standard deviation

\subsection{Consistency of fit procedures}

We have calculated many characteristics of our data. Some of the characteristics can be calculated in different ways. An important point then is to show that the various ways of calculating a particular characteristic lead to the same values.

First, we checked that $\phi_{0}$ in (5) can be put to zero. The proportional reduction in variance brought about by adding $\phi_{0}$ to the fit of the return map was larger than $5 \%$ only in exceptional cases. Adding $\phi_{0}$ to the fit of the histogram was useless because only two parameters could be fitted to it reliably, as we argued in the Methods section. Further, as we will show below, the fit with $\phi_{0}$ set to zero generally reproduced the other observed factors nicely. This agreement in parameter estimates between different procedures was less when we included $\phi_{0}$ in the fit.

Second, we showed that the results of the fit of the return map and the histogram were in agreement. Note that for the histogram we fix one of the parameters to the value from the return map fit (see Methods). For the parameter $a$, agreement, was excellent, with correlations in the range $0.87-0.99$. For the parameter $b$, agreement was even better, with correlations in the range 0.9-0.99. For the parameter $Q$ we find lower correlations in the range $0.69-0.96$. This is caused by an overestimation of the noise by the return map fit when the system is close to the bifurcation. In particular, phase values off the attractor cause this high noise estimate.

Having obtained $a$ and $b$ from the fit, we can calculate the mean phase with $(6,7)$, provided that $|a|<|b|$ (i.e. that there exists a fixed point). The correlations between this predicted mean phase and the measured mean phase for each subject are in the range 0.84-0.99. Likewise, based on $a, b$ and $Q$, we can determine the angular deviation with (9), provided that $|a|<|b|$. The correlation between predicted and measured angular deviation for each subject is in the range 0.82-0.94.

A non-trivial result is that the inverse of the relaxation time correlates significantly with the local stability measure $\pi$. We cannot expect these correlations to be very large because our frequency manipulation has no significant influence on relaxation time (see Fig. 7). We

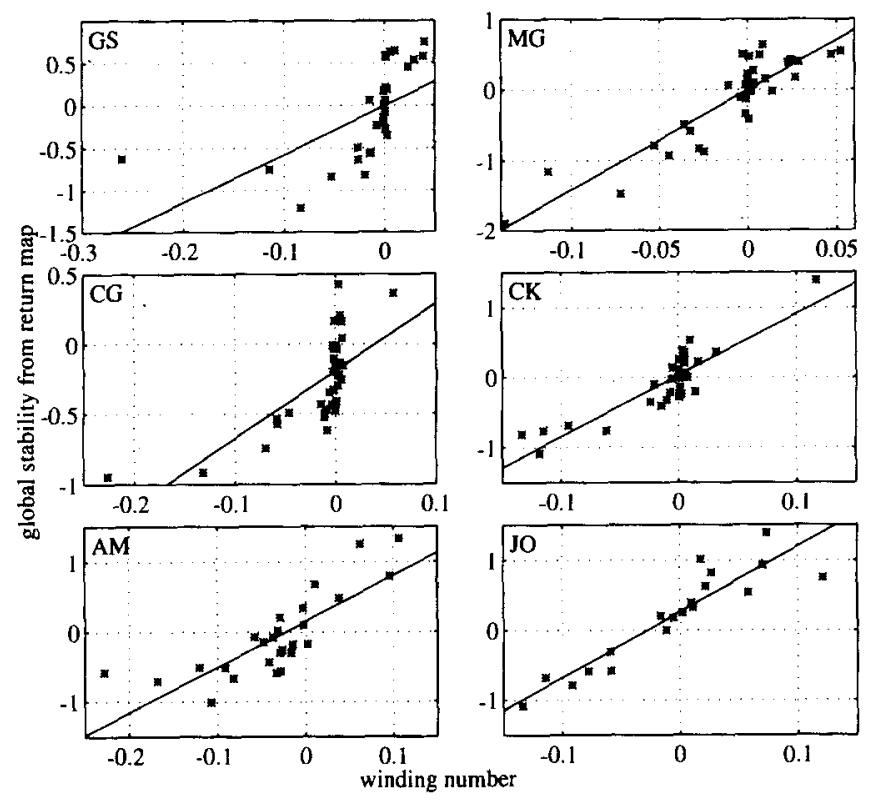

Fig. 11. Scatterplots of the correlation between winding number and the global stability measure $\gamma$ as calculated from the return map fit. The six panels are for six different subjects. Solid lines denote the best fit of a linear regression

find correlations ranging from 0.17 to 0.65 , mean 0.38 . Half of these correlations are significantly different from zero at a significance level of $5 \%$. The connection between $\lambda$, obtained from a fit of the return map and hence incorporating only temporally contiguous phase values, and relaxation time, obtained on the decay of relative phase over multiple time units shows that the fitted dynamics reasonably characterises the dynamic properties of the system.

A final result is that the winding number correlates significantly with the global stability measure $\gamma$. We find correlations ranging from 0.58 to 0.92 , mean 0.8 . All of these correlations are significantly different from zero at a significance level of $5 \%$. For a graphical impression of this correlation, see Fig. 11, which contains scatterplots of the winding number versus $\gamma$ for all subjects. The high correlation is partly caused by the fact that the sign of $a$ correlates highly with the sign of the winding number. One can also see from the plots that the winding number is usually zero for a range of global stability values around zero (especially clear for subjects CG and CK). The relation between winding number and the parameters of the sine-circle map is not known for the noise levels we observed. From simulations we have the impression that the observed correlations are in the range of what can be expected.

\section{Discussion}

We reported the results of experiments on visually induced postural sway. Our focus was on the coordination patterns between stimulus movement and postural response. To characterise this relationship, we measured the relative phase between sway movement and the 
sinusoidal movement of the scene. This provided us with a time series characterising the temporal evolution of this relationship. We manipulated the frequency of the stimulus with the purpose of exploring the limits of a linear model proposed by Schöner (1991). In a previous study (Dijkstra et al. 1994) we varied the distance to the moving wall to test a central prediction of the Schöner model, that temporal stability decreases with increasing visual distance. Generally, we found that the model captured the temporal aspects of the data quite well, but a discrepancy was obtained when the predicted strong decrease of response amplitude was not observed. Our conclusion was that postural sway may be actively generated, and the present experiment aimed to rigorously test this hypothesis.

We obtained evidence for active generation of postural sway at three levels of analysis: (1) the phenomenology of observed coordination patterns, (2) the frequency dependence of measures of temporal order, and (3) the estimated underlying dynamics of relative timing.

In relation to the observed patterns of coordination of sway and visual motion, we found a rich variety of patterns, which can be described using the vocabulary of von Holst (1937/1973). For the middle frequencies $(0.2-0.3 \mathrm{~Hz})$ we observed almost exclusively absolute coordination, i.e. stimulus movement and postural response were phase-locked. This type of coordination was also observed for low $(0.05-0.1 \mathrm{~Hz})$ and high $(0.4-0.5 \mathrm{~Hz})$ frequencies. We found relative coordination, where the stimulus clearly influences the response, but the influence is not sufficiently strong to establish phase-locking, occasionally at higher frequencies. During relative coordination, the system remains locked at relative phase values $\pm \pi / 2$ for periods of time with little systematic change (slow dynamics). Occasionally, locking is lost, and phase wrapping is observed (fast dynamics), until the system is again caught into a locked state. Finally, we observed absence of coordination, where there is no observable influence of the stimulus on the response in terms of relative timing (but in terms of movement amplitude and frequency). This pattern was occasionally observed at low and high frequencies. Both relative coordination and absence of coordination cannot arise in a passively driven system. Such systems (in the stationary state) always respond with the driving frequency and thus are phase-locked. Observing these patterns is therefore evidence that the postural control system is actively generating postural sway.

The dependence of mean phase difference between stimulus and response on frequency was similar to that reported by Lestienne et al. (1977) and van Asten et al. (1988a). In contrast to these studies, much smaller amplitudes of visual motion were used in the present study. Talbott's (1980) study on standing dogs used more comparable amplitudes. These results have conventionally been interpreted as supportive of the notion that the posture control system can be described by a passively driven, second-order, linear system. Our results reveal that this might not be a valid conclusion. Mathematically, driven non-linear oscillators may behave similar to a driven linear system in terms of the mean relative phase.
Hints at non-linear behaviour are obtained from the dependence of the angular deviation of relative phase on frequency. Its increase towards higher and lower frequency points at a decrease of relative timing stability which is not predicted by the Schöner model. Relaxation time did not change with frequency. This would hint at constant stability of relative timing independent of frequency. However, this result as well as the weak correlation of the two stability measures, relaxation time and angular variance of relative phase, could be due to underestimation of relaxation time for states of lower stability. Corroborating evidence for the decrease of stability at higher and lower frequencies comes from the increase in the number of non-locked trials at these frequencies.

We investigated a dynamical model of the relative phase time series based on the sine-circle map. This model contains all observed coordination patterns as qualitatively different solutions: absolute coordination in terms of a stable fixed point, relative coordination when the fixed point attractor is close to a tangent bifurcation, and absence of coordination sufficiently far beyond the bifurcation. We were able to reliably fit the parameters $(a, b$ and $Q$ ) of the sine-circle map both from the return map as well as from the relative phase histogram with convergent results. Moreover, we were able to predict further measurable factors from the estimated dynamics. We found, for instance, that the inverse of relaxation time could be predicted from the local stability measure $\bar{\lambda}$ as obtained from a fit of the return map with reasonable accuracy.

At the level of the estimated model parameters, the fact that these parameters indicate a bifurcation at increasing frequency is evidence for the active nature of the action-perception dynamics. Additional evidence comes from the details of the frequency dependence of the parameters: the parameter $a$, which can be interpreted in terms of the difference between the eigenfrequency of the postural system and the driving frequency, shows roughly the same behaviour as the mean phase. Eigenfrequency is higher than driving frequency at low driving frequencies and the reverse at high driving frequencies. However, if the frequency difference predicted by the linear model is fitted to the model parameter $a$ as obtained at each frequency, a poor fit (around a mean eigenfrequency of $0.2 \mathrm{~Hz}$ ) is obtained. This suggests that the eigenfrequency is not a constant, but that it is adapted to the driving frequency. The parameter $b$, which can be interpreted as the coupling strength between visual motion and postural response, shows an increase in absolute value with frequency.

We have accumulated evidence that there are important deviations from the theoretical picture that we referred to as 'passive' generation of sway. More precisely, passive generation of sway could be defined as a dynamics of sway in which visual motion occurs merely as an additive driving force. In other words, the parameters of the sway dynamics itself are independent of the parameters of visual motion. In the simplest case, the sway dynamics is linear in this theoretical picture. Our evidence suggests instead 'active' generation of sway, in which the sway dynamics reflects the properties of visual 
motion other than through the driving force. This means that parameters such as the frequency and amplitude of visual motion have been built into the postural system (probably through adaptation) such that now the postural system itself generates sway motion, matching properties of the visual motion. This might include nonlinear sway dynamics, in which the linear part of the sway dynamics might be unstable. In this manner, sway can continue to reflect properties of the visual motion in the absence of temporal coherence between sway and visual motion. Active generation of sway in this sense is the only manner in which the posture system can produce a matching amplitude and frequency response over an entire range of frequencies and amplitudes of visual motion. Active generation of sway could thus be viewed as an instance of central nervous adaptation, in which as much information as available is built into the system so as to rely minimally on currently available sensory information.

An interesting open question is why subjects display such a great variety of dynamic behaviours at the extreme frequencies. Some subjects show all three types of coordination for different repetitions of the same frequency condition. We tried to relate the behaviour in a trial to the driving frequency of the preceding trial (testing for some kind of hysteresis) but never found an effect. Presumably, if there is such a form of adaptation at all, it has already taken place in the first $20 \mathrm{~s}$ of each trial which was not recorded. It would be an interesting experiment to switch frequency within a trial and see how subjects adapt. In view of our theoretical results, it is important to keep in mind that near the bifurcation, small changes in the parameters of the dynamics (which might occur spontaneously even at constant frequency in the form of parametric fluctuations) lead to large and even qualitative differences in the resulting solutions. In this respect it is remarkable that even as the types of solutions vary, the estimated model parameters change continuously (cf. Figs. 9 and 10).

In summary, we conclude that temporally structured postural sway is actively generated. The largely periodic temporal structure of postural sway reflects perceived or adaptations to parameters of visual motion. Coupling to visual motion is used to generate phase-locked postural sway that minimises motion relative to the visual world. Phase-locking to visual motion is not, however, a prerequisite to postural sway with a significant amplitude.

Acknowledgements. This work was supported by the Foundation for Biophysics, MUCOM (ESPRIT BRA 3149 and 6615), and by
MWF, Düsseldorf. We would like to thank Erco Argante and Axel Steinhage for their assistance.

\section{References}

Asten WNJC van, Gielen CCAM, Denier van der Gon JJ (1988a) Postural adjustments induced by simulated motion of differently structured environments. Exp Brain Res 73:371-383

Asten WNJC van, Gielen CCAM, Denier van der Gon JJ (1988b) Postural movements induced by rotations of visual scenes. J Opt Soc Am [A] 5:1781-1789

Batschelet E (1981) Circular statistics in biology. Academic Press, London

Bohr T, Bak P, Jensen MH (1984) Transition to chaos by interaction of esonances in dissipative systems. II. Josephson junctions, chargedensity waves, and standard maps. Phys Rev A30:1970-1981

Carter GC (1987) Coherence and time delay estimation. In: Lacoume JL, Durrani TS, Stora R (eds) Signal processing, Les Houches, Session XLV. Elsevier, Amsterdam

Dichgans J, Brandt T (1978) Visual-vestibular interaction: effects on self-motion perception and postural control. In: Held R, Leibowitz HW, Teuber HL (eds) Perception. (Handbook of Sensory Physiology, Vol 8) Springer, Berlin Heidelberg New York

Dijkstra TMH, Schöner G, Gielen CCAM (1994) Temporal stability of the action-perception cycle for postural control in a moving visual environment. Exp Brain Res 97:477-486

Guckenheimer J, Holmes P (1983) Nonlinear oscillations, dynamical systems, and bifurcations of vector fields. Springer, Berlin Heidelberg New York

Holst E von (1937/1973) On the nature of order in the nervous system. In: Martin R (trans) The behavioural physiology of animals and man, Vol 1. Methuen, London

Jensen MH, Bak P, Bohr T (1984) Transition to chaos by interaction of resonances in dissipative systems. I. Circle maps. Phys Rev A30:1960-1969

Kelso JAS, Delcolle JD, Schöner G (1990) Action-perception as a pattern formation process. In: Jeannerod $\mathrm{M}$ (ed) Attention and Performance XIII. Erlbaum, Hillsdale, pp 139-169

Lee DN, Lishman JR (1975) Visual proprioceptive control of stance. J Hum Mov Studies 1:87-95

Lestienne F, Soechting J, Berthoz A (1977) Postural readjustments induced by linear motion of visual scenes. Exp Brain Res 28:363-384

Marple SL (1987) Digital spectral analysis with applications. Prentice Hall, Englewood Cliffs

Press WH, Flannery BP, Teukolsky SA, Vetterling WT (1988) Numerical recipes in C. Cambridge University Press, Cambridge, UK

Reichl LE (1980) A modern course in statistical mechanics. Edward Arnold, London

Schöner G (1991) Dynamic theory of action-perception patterns: the "moving room" paradigm. Biol Cybern 64:455-462

Schöner G, Kelso JAS (1988) Dynamic pattern generation in behavioural and neural systems. Science 239:1513-1520

Schuster D, Talbott RE (1980) Optimal and adaptive control in canine postural regulation. Am J Physiol 239:R93-R114

Talbott RE (1980) Postural reactions of dogs to sinusoidal motion in the peripheral visual field. Am J Physiol 239:R71-R79

Talbott RE, Brookhart RE (1980) A predictive model study of the visual contribution to canine postural control. Am J Physiol 239: R80-R92

Wiesenfeld K, Satija I (1987) Noise tolerance of frequency-locked dynamics. Phys Rev B36:2483-2491 